\title{
The wearable cardioverter defibrillator: Still without a compelling indication
}

\author{
Peter Ott* \\ Clinical Medicine, Sarver Heart Center, Tucson, Arizona, USA
}

The wearable cardioverter defibrillator (WCD) has recently been introduced as a therapy to reduce mortality in patients presumed to be at increased risk for sudden arrhythmic death. Its efficacy appears to be implied by its proven ability to detect and defibrillate ventricular tachycardia/ventricular fibrillation (VT/VF). However, the currently available data on the WCD is non-randomized and comes from several reports of single center experiences, and reviews of post market release, often vendor supported, registry data. In the absence of randomized trials, the effect of the WCD on patient survival cannot be determined. The mere ability to shock terminate VT/VF by the WCD does not suffice to assume a reduction in mortality when used in large patient populations. These severe limitations have been acknowledged in the recent AHA science advisory report [1], providing a "tentative interim framework" resulting in mostly class II b recommendations ("treatment may be considered") with only level C support ("consensus opinion by experts only"). This contrast sharply with AHA class I recommendations ("treatment should be considered") with level A support ("multiple clinical randomized trials") for the implantable cardioverter defibrillator (ICD).

The WCD is being heavily advertised to temporarily "protect" patients presumed at increased risk for arrhythmic death (primary prevention), in particular patients early after acute myocardial infarction (MI) or with newly diagnosed dilated cardiomyopathy. In these patient populations, several randomized trials with the implantable ICD [2-4] failed to show mortality reduction and these patients remain temporarily ineligible for device implantation for a period of 40 days (early after acute MI) or 90 days (newly diagnosed dilated cardiomyopathy). In practical terms, these time periods serve to allow for optimizing medical therapy, which often results in improvement of cardiac function with subsequent removal from ICD eligibility. The actual mortality risk, and in particular the risk of sudden arrhythmic death, and thus the utility of the WCD during these waiting periods is not well established.

Clinical research has shown an increased risk of sudden death in patients early after acute MI. One of the largest trials, addressing this clinical issue, was the VALIANT trial [5], it randomized 14,609 patients after acute MI with reduced LV function and/or clinical heart failure to valsartan, captopril, or both. During 2.4 year follow up, 2,800 deaths were observed, of which 1067 were classified as either sudden death $(\mathrm{N}=903)$ or resuscitated cardiac arrest $(\mathrm{n}=104)$. The median time to event occurrence was 180 days, but 580 events occurred within the first 30 days (event rate $1.4 \%$ ), suggesting a possible role for defibrillator therapy early after acute MI. However, two trials [2,3] in a total of 1,572 patients early after acute MI with reduced LV function and additional risk markers, randomized to medical therapy with or without ICD implantation, failed to show a reduction in overall mortality. While in both trials the arrhythmic mortality was reduced in the ICD group, an increase on non-arrhythmic mortality was seen resulting no net benefit in total survival. VALIANT trial autopsy data [6] on 398 patients, 105 were classified clinically as sudden, presumed arrhythmic, death, revealed a non-arrhythmic mechanism (mostly recurrent MI or cardiac rupture) in $51 \%$. This may explain, at least in part, the lack of benefit from ICD therapy in these trials.

Information on the WCD in the immediate post MI period is based primarily on a large post market release database review $(\mathrm{n}=$ 8,453 ), with, by design, limited clinical information [7]. In the first three months 133 patients (1.6\%) were defibrillated by the WCD for ventricular arrhythmias. Despite successful defibrillation, 51 (38\%) of those patients died (12 patients did not survive to ER, 3 died within 2 days after admission and 41 died more than 3 days after the shock event). Sustained ventricular arrhythmias are not necessarily lethal. Indeed, in the WEARIT-II registry $(\mathrm{n}=2,000), 41$ patients experienced $120 \mathrm{VT} / \mathrm{VF}$ events during a 3-month follow. For $90 \mathrm{VT}$ events, the patient withheld WCD therapy due to hemodynamic tolerance [8]. Thus, in patients early after acute MI, the effect of the WCD on total mortality remains unknown.

Patients with newly diagnosed dilated cardiomyopathy may be at risk for sudden arrhythmic death. The only randomized trial comparing ICD with no ICD therapy in patients with recent onset ( $<9$ months) non-ischemic cardiomyopathy on optimal medical therapy was terminated early due to low overall mortality and lack of survival benefit [4]. Likewise, recent registry data on the WCD use in this patient population has shown a very low incidence of device treated VT/VF: in the initial nationwide registry data [9] only 4 out of 546 patients with newly diagnosed DCMP experienced VT/VF events defibrillated by the WCD during a 2 month follow up. The more recent prospective WEARIT-II registry [8] showed a 1\% VT/VF event rate in 922 patients with newly diagnosed DCMP within 3 months of WCD therapy. A single center experience [10] reported no shocks for VT/VF in 271 patients with newly diagnosed non-ischemic cardiomyopathy during a median wear time of 71 days. Reversibility of the cardiomyopathy processes, as well as early improvement in LV function with vigorous pharmacological therapy, may explain the low incidence of VT/VF in this patient population, and suggests that the addition of short-term therapy with the WCD may have limited, if any, effect on overall survival.

Correspondence to: Peter Ott, Clinical Medicine, Sarver Heart Center, BANNER UMC, Tucson, Arizona, USA, Tel: 520-626-6358; Fax: 520-626-4333; E-mail: pott@shc.arizona.edu

Received: March 21, 2017; Accepted: April 21, 2017; Published: April 24, 2017 
The lack of clinical randomized trial data of the effect of the WCD on mortality in these particular patient populations is concerning. Given the risk of sudden death, some no doubt arrhythmic in nature, yet absence of mortality benefit with standard ICD technology, enrolment in a randomized trial using optimal medical therapy with or without the non-invasive WCD would be expected to be brisk. The trial would focus on total mortality as the primary endpoint, during a short follow-up period of 1-3 months (40-90 day waiting period); results would be expected quickly. However, the only randomized trial with the WCD (VEST, NCT01446985), started enrolling patients with LV ejection fraction $<35 \%$ early after acute MI in 2008. The goal is to enroll 1,900 patients. The primary outcome is sudden cardiac death at 3 months after MI, and results are not expected until 2017.

Psychological stressors are well known to trigger ventricular arrhythmias for example after natural disasters [11] or terror attacks [12]. Wearing a WCD may cause anxiety and psychological distress. Indeed, in a recent European survey [13], anxiety and fear from WCD shock, as well as significant sleep disturbances were reported by almost half of patients. While data from registries report high rates of compliance in wearing the WCD, its psychological impact, and thus its potential for pro-arrhythmic effects, is unknown and can only be evaluated in a randomized trial.

Thus, in patients with reduced LV function early after acute MI or new onset dilated cardiomyopathy, the data presented above shows low VT/VF event rates, a substantial proportion of non-arrhythmic sudden death mechanism on autopsy, hemodynamic tolerance of many VT episodes and a significant short-term mortality despite successful VT/ VF termination. Thus, there is doubt as to whether short-term therapy with the WCD would reduce mortality in these patients. A potential for pro-arrhythmic effect related to anxiety/stress while wearing the WCD has not been evaluated. Results of randomized studies of WCD therapy as primary prevention of sudden death in these patient populations are urgently needed before widespread use of this technology can be recommended.

\section{References}

1. Piccini JP Sr, Allen LA, Kudenchuk PJ, Page RL, Patel MR, et al. (2016) Wearable
Cardioverter-Defibrillator Therapy for the Prevention of Sudden Cardiac Death: A Science Advisory From the American Heart Association. Circulation 133: 1715-1727. [Crossref]

2. Hohnloser SH, Kuck KH, Dorian P, Roberts RS, Hampton JR, et al. (2004) Prophylactic use of an implantable cardioverter-defibrillator after acute myocardial infarction. $N$ Engl J Med 351: 2481-2488. [Crossref]

3. Steinbeck G, Andresen D, Seidl K, Brachmann J, Hoffmann E, et al. (2009) Defibrillator implantation early after myocardial infarction. N Engl J Med 361: 14271436. [Crossref]

4. Bänsch D, Antz M, Boczor S, Volkmer M, Tebbenjohanns J, et al. (2002) Primary prevention of sudden cardiac death in idiopathic dilated cardiomyopathy: the Cardiomyopathy Trial (CAT). Circulation 105: 1453-1458. [Crossref]

5. Solomon SD, Zelenkofske S, McMurray JJ, Finn PV, Velazquez E, et al. (2005) Sudden death in patients with myocardial infarction and left ventricular dysfunction, heart failure, or both. $N$ Engl J Med 352: 2581-2588. [Crossref]

6. Pouleur AC, Barkoudah E, Uno H, Skali H, Finn PV, et al. (2010) Pathogenesis of sudden unexpected death in a clinical trial of patients with myocardial infarction and left ventricular dysfunction, heart failure, or both. Circulation 122: 597-602. [Crossref]

7. Epstein AE, Abraham WT, Bianco NR, Kern KB, Mirro M, et al. (2013) Wearable cardioverter-defibrillator use in patients perceived to be at high risk early postmyocardial infarction. J Am Coll Cardiol 62: 2000-2007. [Crossref]

8. Kutyifa V, Moss AJ, Klein H, Biton Y, McNitt S, et al. (2015) Use of the Wearable Cardioverter Defibrillator in high-risk cardiac patients. Data From the Prospective Registry of Patients Using the Wearable Cardioverter Defibrillator (WEARIT-II Registry). Circulation 132: 1613-1619. [Crossref]

9. Chung MK, Szymkiewicz SJ, Shao M, Zishiri E, Niebauer MJ, et al. (2010) Aggregate national experience with the wearable cardioverter-defibrillator: event rates, compliance, and survival. J Am Coll Cardiol 56: 194-203. [Crossref]

10. Singh M, Wang NC, Jain S, Voigt AH, Saba S, et al. (2015) Utility of the Wearable Cardioverter-Defibrillator in Patients With Newly Diagnosed Cardiomyopathy: A Decade-Long Single-Center Experience. J Am Coll Cardiol 66: 2607-2613. [Crossref]

11. Leor J, Poole WK, Kloner RA (1996) Sudden cardiac death triggered by an earthquake. N Engl J Med 334: 413-419. [Crossref]

12. Steinberg JS, Arshad A, Kowalski M, Kukar A, Suma V, et al. (2004) Increased incidence of life-threatening ventricular arrhythmias in implantable defibrillator patients after the World Trade Center attack. J Am Coll Cardiol 44: 1261-1264. [Crossref]

13. Lenarczyk R, Potpara TS, Haugaa KH, Hernández-Madrid A, Sciaraffia E, et al. (2016) The use of wearable cardioverter-defibrillators in Europe: results of the European Heart Rhythm Association survey. Europace 18: 146-150. [Crossref]

Copyright: (C2017 Ott P. This is an open-access article distributed under the terms of the Creative Commons Attribution License, which permits unrestricted use, distribution, and reproduction in any medium, provided the original author and source are credited. 